\title{
Lower versus upper leg saphenous vein composite grafts based on the left internal thoracic artery: A randomized study
}

\author{
Hyoung Woo Chang, MD, PhD, ${ }^{a}$ Ho Young Hwang, MD, PhD, ${ }^{\mathrm{b}}$ Jeong-Wook Seo, MD, PhD, ${ }^{\mathrm{c}}$ and \\ Ki-Bong Kim, $\mathrm{MD}, \mathrm{PhD}^{\mathrm{b}}$
}

\section{ABSTRACT}

Objectives: The "lower versus upper leg saphenous vein (SV) composite graft based on the left internal thoracic artery (ITA) for coronary artery bypass grafting" trial was designed to compare the histologic, immunohistochemical, and angiographic findings of lower versus upper leg SV composite grafts.

Methods: Twenty-six patients with multivessel coronary artery disease were prospectively randomized to undergo revascularization using a lower leg $(n=13)$ or upper leg $(n=13)$ SV composite graft based on the in situ left ITA. The SV was harvested with a "no-touch" technique, and 2 excess segments were removed from the distal and proximal portions of each SV conduit. Another proximal segment was removed from the reversed SV composite graft, which had been dilated by the native ITA pressure. Hematoxylin and eosin staining, immunohistochemistry, and early and 1-year postoperative angiographic results were compared.

Results: The histologic study showed that the proximal and dilated proximal SV conduit lumen diameters were smaller in the lower leg group than in the upper leg group (proximal, $623 \pm 143 \mu \mathrm{m}$ vs $858 \pm 266 \mu \mathrm{m} ; P=.008$; dilated proximal, $1138 \pm 419 \mu \mathrm{m}$ vs $1477 \pm 353 \mu \mathrm{m} ; P=.047)$. However, there were no differences in the lumen diameters of the distal SV segments in terms of immunohistochemical comparisons, diameters, patency rates, or filling frame counts of the SV conduits on early and 1-year postoperative angiograms between the 2 groups.

Conclusions: Although the proximal segment luminal diameters were smaller in the lower leg SV, there were no differences in the immunohistochemical results or patency rates on early and 1-year postoperative angiograms between the lower and upper leg "no-touch" SV conduits. (J Thorac Cardiovasc Surg 2018;156:986-94)

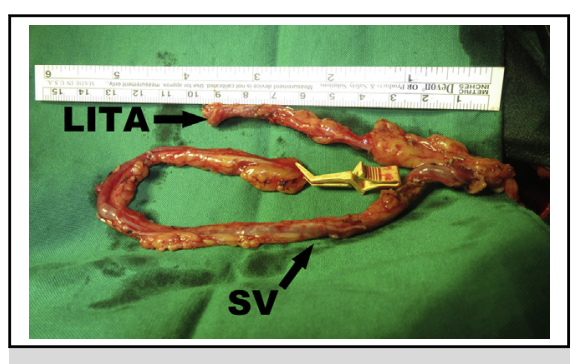

Composite graft (left internal thoracic artery and "notouch" saphenous vein)

\section{Central Message}

No statistically significant difference was found in the immunohistochemistry or in the early and 1-year postoperative patency rates between the lower and upper leg "no-touch" saphenous vein conduits.

\section{Perspective}

No statistically significant difference was found in the immunohistochemical results or the 1year patency rates between the lower and upper leg "no-touch" saphenous vein conduits, suggesting uncertain advantage in using either one of the saphenous veins as a conduit in coronary artery bypass graft surgery.

See Editorial Commentary page 995.
The great saphenous vein $(\mathrm{SV})$ is the longest vein in the human body and remains a widely used conduit of choice in coronary artery bypass graft surgery (CABG), although lower long-term patency rates and worse clinical outcomes have been reported after $\mathrm{CABG}$ performed with SV conduits than after $\mathrm{CABG}$ performed with arterial conduits. ${ }^{1-3}$

From the ${ }^{\mathrm{a}}$ Department of Thoracic and Cardiovascular Surgery, Sejong General Hospital, Bucheon-si, Gyeonggi-do, Republic of Korea; and Departments of ${ }^{\mathrm{b}}$ Thoracic and Cardiovascular Surgery and ${ }^{\mathrm{c}}$ Pathology, Seoul National University Hospital, Seoul National University College of Medicine, Seoul, Republic of Korea.

This study was supported by a grant from Seoul National University Hospital (grant number 0420130260).

Received for publication July 17, 2017; revisions received Jan 8, 2018; accepted for publication March 2, 2018; available ahead of print June 5, 2018.

Address for reprints: Ki-Bong Kim, MD, PhD, Department of Thoracic and Cardiovascular Surgery, Seoul National University Hospital, 101 Daehak-ro, Jongno-gu, Seoul 03080, Republic of Korea (E-mail: kimkb@snu.ac.kr).

$0022-5223 / \$ 36.00$

Copyright (c) 2018 by The American Association for Thoracic Surgery

https://doi.org/10.1016/j.jtcvs.2018.03.148
Recent studies have suggested that using a minimally manipulated SV as a composite graft based on the in situ left internal thoracic artery (ITA) could overcome the limitations of SV grafts. ${ }^{4-6}$ In addition, a "no-touch" SV harvesting technique, in which the vein is harvested with a pedicle of surrounding tissue, has been suggested to further improve the SV patency by increasing the preservation of vessel integrity.

The great SV runs from the ankle to the region of the femoral triangle, and there are differences in vessel diameter and localization between the lower and upper leg SV. However, few studies have evaluated the functional or

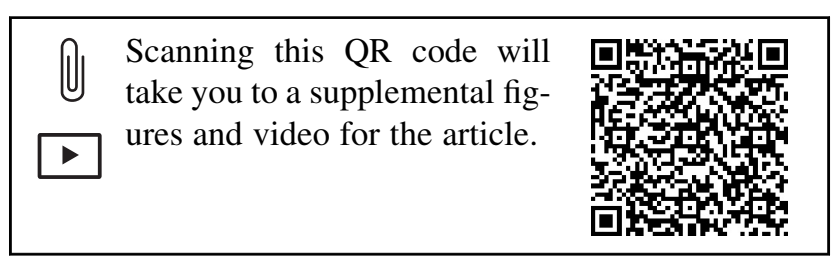



Abbreviations and Acronyms
$\mathrm{CABG}=$ coronary artery bypass graft surgery
ITA $=$ internal thoracic artery
KLF4 = Krüppel-like factor 4
LLV = lower leg vein
SMD $=$ standardized mean difference
$\mathrm{SRF}=$ serum response factor
$\mathrm{SV}=$ saphenous vein
$\mathrm{ULV}=$ upper leg vein
VSMC $=$ vascular smooth muscle cell

morphologic differences between the lower and upper leg $\mathrm{SV},{ }^{9-11}$ and the choice of the lower versus upper leg SV as a conduit in CABG has been the surgeon's preference. The aim of this study was to compare the histologic and immunohistochemical differences and the early and 1-year postoperative angiographic results between the lower and upper leg SV harvested with a "no-touch" technique and used as composite grafts based on the in situ left ITA.

\section{METHODS \\ Study Design}

The study was designed according to the Consolidated Standards of Reporting Trials statement. ${ }^{12}$ This study, the Lower versus Upper leg saphenous vein coMposite graft based on the $1 \mathrm{Eft}$ internal thoracic artery for coroNary artery bypass grafting (LUMEN) trial, was a randomized, controlled, open-label clinical trial. The institutional review board approved the study protocol on October 25, 2013 (approval number $\mathrm{H}-1308-077-514)$, and all of the study patients provided informed consent (ClinicalTrials.gov identifier, NCT01974492).

Of 50 patients who underwent first-time isolated $C A B G$ at our institution between November 2013 and February 2014, 26 patients were eligible for this study because they met the following criteria: 40 to 75 years of age, scheduled to undergo CABG for multivessel coronary artery disease on a nonemergency basis, and expected to receive a Y-composite graft based on the in situ left ITA for complete revascularization. The eligible patients $(\mathrm{n}=26)$ were randomly assigned in a 1:1 manner to 1 of the 2 surgical strategies on the basis of the side-arm conduit used to construct the Y-composite graft: the lower leg SV Y-composite graft (group LLV, $\mathrm{n}=13$ ) or the upper leg SV Y-composite graft (group ULV, $\mathrm{n}=13$; Figure 1). The exclusion criteria included patients with an unavailable $\mathrm{SV}$, a history of a previous cardiac surgery, or a medical history that might limit the possibility of midterm follow-up, such as malignant disease. Patients with an estimated left ventricular ejection fraction $\leq 25 \%$ were also excluded because of the possibility of the need for an additional conduit in a dilated left ventricle.

After the pericardium was opened and the coronary arterial anatomies had been assessed for availability for revascularization using a Y-composite graft, randomization was performed using a block randomization method stratified according to patient age (40-60 years old vs 61-75 years old) and sex. The table for randomization was provided by the Clinical Research Institute of Seoul National University Hospital, and a doctor who was not involved in this study informed the surgeon of the harvesting site (lower vs upper leg). There were no statistically significant differences in the demographic data or preoperative risk factors between the 2 groups (Table 1).

\section{Surgical Strategies}

All of the patients underwent off-pump CABG during the study period, and the basic surgical procedures and strategies for off-pump CABG and the technique for harvesting the SV have been previously described. ${ }^{5,8}$

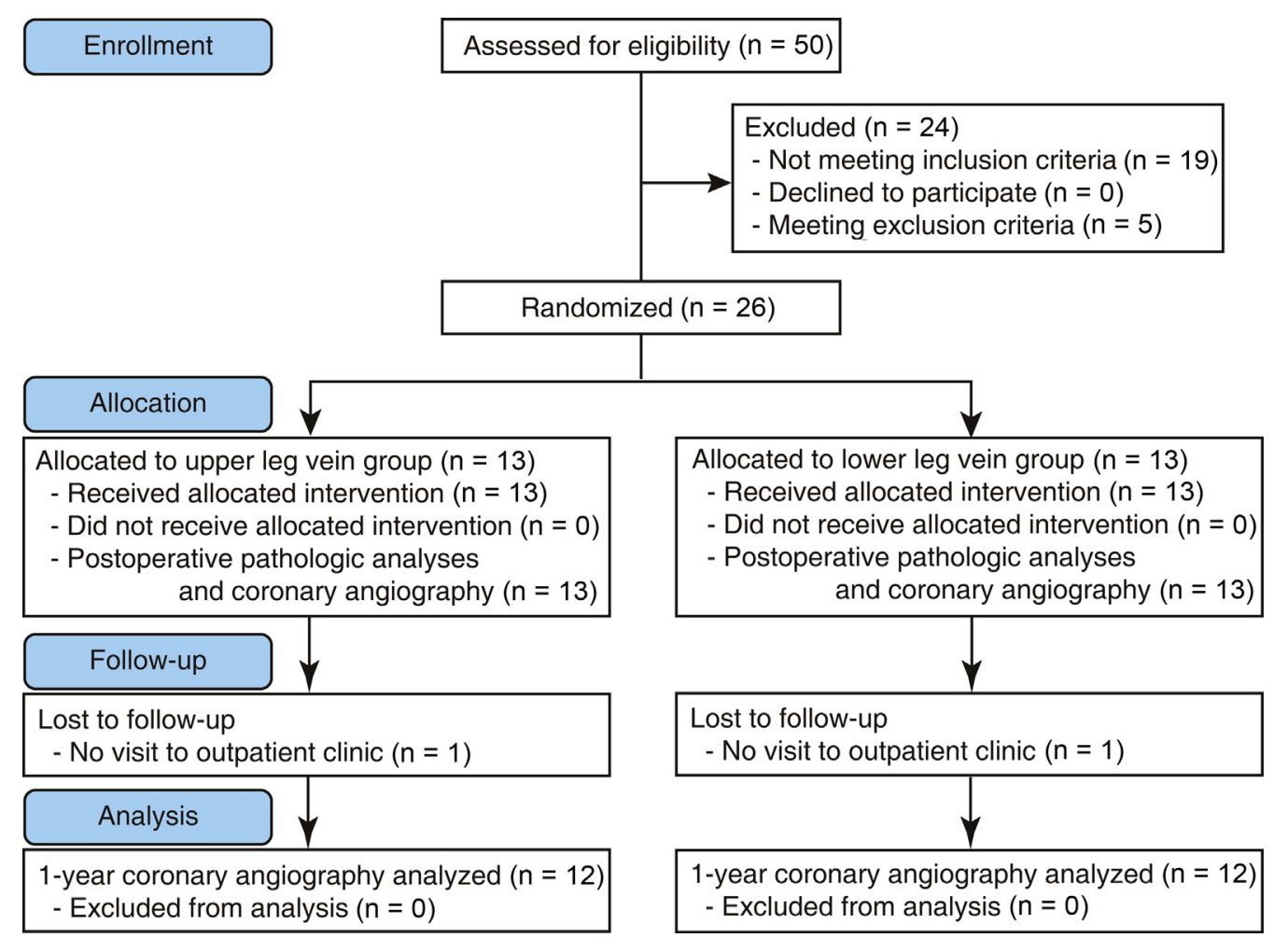

FIGURE 1. Consolidated Standards of Reporting Trials flow diagram. 
TABLE 1. Preoperative characteristics and risk factors of the study patients

\begin{tabular}{|c|c|c|c|c|}
\hline & Group LLV $(n=13)$ & Group ULV $(n=13)$ & $P$ value & SMD \\
\hline Mean age $\pm S D, y$ & $63 \pm 7$ & $66 \pm 7$ & $.486^{*}$ & 0.33 \\
\hline Female sex & $3(23 \%)$ & $3(23 \%)$ & $>.999 \dagger$ & $<0.01$ \\
\hline \multicolumn{5}{|l|}{ Risk factors, $(\%)$} \\
\hline Smoking & $6(46)$ & $3(23)$ & $.411 \dagger$ & 0.48 \\
\hline Hypertension & $5(38)$ & $9(69)$ & $.238 \dagger$ & 0.62 \\
\hline Diabetes mellitus & $8(62)$ & $5(38)$ & $.434 \dagger$ & 0.46 \\
\hline Dyslipidemia & $5(38)$ & $1(8)$ & $.160 \dagger$ & 0.75 \\
\hline History of stroke & $1(8)$ & $2(15)$ & $>.999 \dagger$ & 0.23 \\
\hline Chronic renal failure & $2(15)$ & $2(15)$ & $>.999 \dagger$ & $<0.01$ \\
\hline Left main disease & $10(77)$ & $5(38)$ & $.111 \dagger$ & 0.81 \\
\hline Preoperative diagnosis, $(\%)$ & & & $.265 \dagger$ & \\
\hline Stable angina & $7(54)$ & $6(46)$ & & \\
\hline Unstable angina & $4(31)$ & $7(54)$ & & \\
\hline Postinfarction angina & $2(15)$ & $0(0)$ & & \\
\hline
\end{tabular}

$L L V$, Lower leg saphenous vein; $U L V$, upper leg saphenous vein; $S M D$, standardized mean difference. *Mann-Whitney $U$ test. $\dagger$ Fisher exact test.

SV harvesting was initiated after systemic heparinization therapy and was performed using an open technique. After anesthetic induction, Doppler ultrasonography mapping was performed to assess the course and quality of the SV. The left leg SV was preferred because harvesting from the left side of the patient enabled easy simultaneous harvesting of the left ITA from the right side of the patient. If ultrasonography mapping revealed any abnormal findings, such as too-small caliber, ectatic change, or thrombotic occlusion, the right leg SV was used instead. Patients were administered an initial dose of heparin $(1.5 \mathrm{mg} / \mathrm{kg})$ and periodic supplemental doses to maintain an activated clotting time of $\geq 300$ seconds. SV harvesting was performed using the "no-touch" technique, ${ }^{13}$ whereby the SV pedicle was harvested along with an approximately 3- to 5-mm wide margin of adjacent adipose tissues on both sides of the SV and thin layers of adherent connective tissues posteriorly. Manipulation of the SV was minimized, and manual intraluminal dilatation was avoided (Figure 2, A). Side branches were divided after metal clipping, and to prevent inadvertent endothelial injury, clips were applied at least $1 \mathrm{~mm}$ from branch origins. Immediately after harvesting, the reversed SV was anastomosed to the left ITA to construct a Y-composite graft without any pharmacologic treatment. All of the diseased vessels with significant ( $\geq 70 \%$ diameter) stenoses were revascularized. Complete revascularization, defined as at least $1 \mathrm{graft}$ to each of the diseased major coronary artery territories, was achieved whenever possible, except in patients with target vessels that were too small $(<1 \mathrm{~mm}$ diameter). The left anterior descending coronary artery territory was revascularized first using the left ITA, whereas the distal end of the SV conduit was dilated spontaneously by the native flow and pressure of the left ITA. The left circumflex coronary artery territory was then revascularized, followed by the right coronary artery territory. A sequential anastomotic technique, using each side arm of the Y-composite graft, was used for complete revascularization when more than 2 coronary arterial anastomoses were required. An intraoperative graft assessment using transit-time flow measurement (Medi-Stim AS, Oslo, Norway) was performed immediately before sternal closure. The mean blood pressure was maintained at between 80 and $90 \mathrm{~mm} \mathrm{Hg}$ during the flow measurement, and a properly fitted probe $(2,3$, or $4 \mathrm{~mm})$ was used to measure the flow. The mean flows of the left ITA 2 to $3 \mathrm{~cm}$ proximal to the $\mathrm{Y}$-anastomosis and the $\mathrm{SV}$ conduit 2 to $3 \mathrm{~cm}$ distal to the Y-anastomosis were recorded and stored in the flow meter. After protamine administration to normalize the prolonged activated clotting time at the end of the off-pump CABG, a Jackson-Pratt drain was inserted in the SV harvesting site, and the leg wounds were closed. The Jackson-Pratt drain was removed when drainage had decreased to $<10 \mathrm{~mL}$ daily.
All of the patients received aspirin therapy (100 mg daily) until the day of surgery and resumed the therapy as soon as possible after surgery, usually 1 day postoperatively. Clopidogrel ( $75 \mathrm{mg}$ daily) was combined simultaneously with aspirin for 1 year postoperatively. If the patient had a high blood level of low-density lipoprotein cholesterol (>100 mg/dL), drug therapy was initiated.

\section{Preparation of the Saphenous Vein for Histological Evaluation}

In the LLV group, proximal dissection of the SV was performed to approximately $3 \mathrm{~cm}$ below the knee, and the SV was separated distally approximately $5 \mathrm{~cm}$ proximal to the ankle. In the ULV group, proximal dissection of the SV was performed to approximately $5 \mathrm{~cm}$ below the region of the femoral triangle, and the SV was divided distally approximately $3 \mathrm{~cm}$ proximal to the knee. Of the 18.0 to $25.5 \mathrm{~cm}$ of SV harvested for grafting, approximately $3 \mathrm{~cm}$ of excess SV were used for this study. The procedure used for the SV sample collection procedure is shown in Figure 2, B. Two 1-cm segments from this 3-cm region were removed from the "distal" and "proximal" SV segments before constructing the Y-composite graft (Figure 2, B). After the Y-composite graft was constructed, the distal end of the SV was clamped with an atraumatic bulldog clamp (Scanlan International Inc, St Paul, Minn), leaving the SV portion of the graft exposed to the native blood flow and pressure of the in situ left ITA for dilation during the distal anastomoses. Immediately before performing the far distal anastomosis, another $1-\mathrm{cm}$ dilated segment of the reversed SV conduit ("dilated proximal" segment) was removed and prepared for this study.

All of the samples were fixed with $10 \%$ formalin solution, embedded in paraffin, and cut into 5- $\mu \mathrm{m}$ thick sections. Hematoxylin and eosin staining was performed to evaluate the SV endothelial integrity. Immunohistochemistry was performed using mouse monoclonal antibodies (Abcam, Cambridge, United Kingdom) against Krüppel-like factor 4 (KLF4), serum response factor (SRF), and myocardin, which are known to regulate the change to a contractile phenotype. KLF4 and SRF were used to evaluate the expression of vascular smooth muscle cell (VSMC)-related molecules, and myocardin was used to evaluate the preservation of medial smooth muscle folding. ${ }^{14}$ The degree of endothelial integrity, expression of VSMC activation-related proteins, and preservation of medial smooth muscle folding were examined in the proximal and dilated proximal segments, which were segments adjacent to each other. 

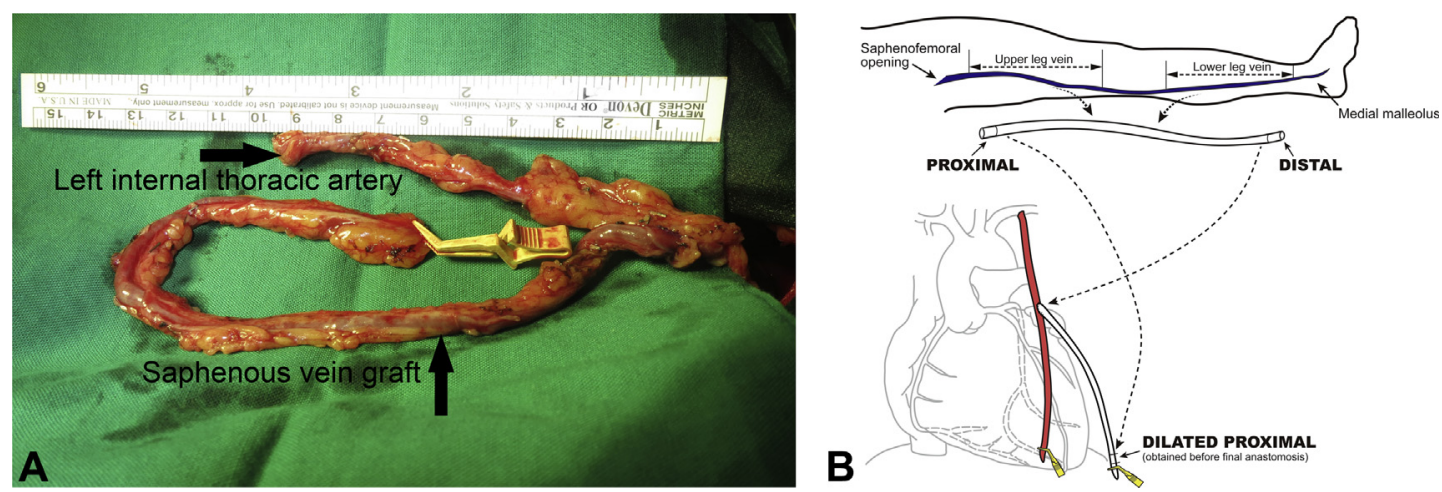

FIGURE 2. A, "No-touch" saphenous vein (SV) composite graft on the basis of the in situ left internal thoracic artery. B, Preparation of 3 SV segments. Two 1-cm segments were removed from the "distal" and "proximal" SV segments before construction of the composite graft, and another 1-cm segment of the reversed SV conduit ("dilated proximal" segment) was prepared immediately before performing the far distal anastomosis.

The endogenous peroxidase activity of the sections was blocked by a 10 -minute preincubation with $3 \%$ hydrogen peroxide in methanol. The sections were then incubated in 5\% normal goat serum in Tris buffer solution at room temperature for 10 minutes to prevent background staining. The sections were then treated with mouse monoclonal antibodies against KLF4, SRF, and myocardin. The sections were rinsed, and secondary antibody conjugated with horseradish peroxidase was applied for 30 minutes. After staining with the diaminobenzidine chromogen for 10 minutes and counterstaining with Meyer's hematoxylin for 15 seconds, the sections were prepared for microscopic examination. The proportion of positive staining in the luminal circumference was graded from 0 to 1 , and the value was rounded to the second decimal place (Figure E1). When the colored tagged molecules were widely displayed along the complete circumference of the intima, the sample was graded as 1.00 . When the molecules' expression was infrequent, the grading value was obtained by measuring the length of the intima in which the molecule appeared and dividing it by the length of the whole intimal inner circumference. The evaluation of medial smooth muscle folding using myocardin followed the same logic. A BX51 optical microscope (Olympus Corp, Tokyo, Japan) was used for the microscopic examination, and the internal luminal diameter as well as the intimal and medial thicknesses were measured using a U-OCM10/ 100 eyepiece micrometer (Olympus Corp) and a stage micrometer (Narika Corp, Tokyo, Japan; Figure E2).

\section{Angiographic Evaluation of Conduits}

Early and 1-year postoperative coronary angiograms to evaluate the grafts were obtained on the basis of the patient's consent, regardless of angina symptoms. Early postoperative (postoperative day $1.1 \pm 0.3$ ) angiography was performed in all study patients (26 of 26). At 1 year (12.8 \pm 1.5 months) postoperatively, $92.3 \%$ of the patients ( 24 of 26 ) underwent graft evaluation using coronary angiograms. One patient from the LLV group who refused angiographic evaluation and 1 patient from the ULV group who died of sepsis after colon surgery during the follow-up period were excluded from the 1-year angiographic follow-up.

After the diagnostic angiography, the left ITA was engaged using a 4- or 5-French guiding catheter to perform the graft angiography, and reference images were acquired after the administration of 100 to $200 \mu \mathrm{g}$ of nitroglycerin. A single experienced observer measured the lumen diameters of conduits using the guiding catheter for calibration and the scientific image analysis software ImageJ (National Institutes of Health, Bethesda, Md). The left ITA diameters were measured at 3 points $(2,4$, and $6 \mathrm{~cm}$ distal to the left ITA origin), and the average value was calculated. The diameters of the $S V$ conduits were also measured at 3 points $(2,4$, and $6 \mathrm{~cm}$ distal to the Y-anastomosis), and the average value was calculated
(Figure E3). The flow velocity was measured using the filling frame count method; the number of angiography video frames required to visualize the $\mathrm{SV}$ from the Y-anastomosis to the far distal anastomosis site of the SV conduit was counted.

\section{Statistical Analysis}

Values for the sample size calculation were drawn from our previous study, ${ }^{4}$ and the sample size was calculated using PASS software, version 11 (NCSS, Kaysville, Utah). Calculations were performed to evaluate the inequality of the degree of luminal staining. To achieve $82 \%$ power to detect a difference of 0.2 between the null hypothesis ("mean values in both groups were 0.9 ") and the alternative hypothesis ("mean values between the 2 groups were significantly different") with an estimated SD of 0.17 in each group and a significance level of 0.05 using a 2-sided 2sample $t$ test, 12 patients in each group were required to complete the study cohort. Considering an approximately $10 \%$ dropout during the 1 -year follow-up period, we determined that recruiting 13 patients in each group was necessary. The sample size for the primary end point were calculated by estimating the general measurement results because previous comparable data were not available for the primary end point, the expression of VSMC activation-related molecules. Statistical analysis was performed using SPSS version 20 (IBM Corp, Armonk, NY) software and SAS version 9.3 (SAS Institute, Inc, Cary, NC) software at the Medical Research Collaboration Center, Seoul National University Hospital. Comparisons between the 2 groups were performed using Fisher exact test for categorical variables and the Mann-Whitney $U$ test for continuous variables. Intragroup pairwise comparisons were done with Wilcoxon signed rank test for continuous variables. The standardized mean differences (SMDs) between the LLV and ULV groups were used for balance assessment between groups in baseline characteristics as well as for group effect size of outcome variables, which was calculated as the mean difference between groups divided by the square root of the average sample variances in the 2 groups. For group effect size, the SMDs of $0.2,0.5$, and 0.8 were considered as small, medium, and large effect sizes, respectively. ${ }^{15}$ Comparisons of the early and 1-year postoperative angiographic results were performed using an analysis of covariance, adjusting for early postoperative vein diameters. $P$ values were calculated on the basis of a 2 -sided test, and a $P$ value $<.05$ was considered statistically significant.

\section{RESULTS}

\section{Surgical Data}

The average length of the harvested SV was $22.3 \pm 1.9 \mathrm{~cm}$ (range, 18.0-25.5 cm; groups LLV vs ULV, 
$22.1 \pm 1.9 \mathrm{~cm}$ vs $22.4 \pm 2.0 \mathrm{~cm} ; P>.999$ ), and the average length of the SV conduit used for anastomoses was $18.0 \pm 1.9 \mathrm{~cm}$ (range, $14.0-21.5 \mathrm{~cm}$; groups LLV vs ULV, $17.6 \pm 1.8 \mathrm{~cm}$ vs $18.4 \pm 2.0 \mathrm{~cm}, P>.999)$. The average number of distal anastomoses per patient, the average number of distal anastomoses using the SV, and the number of distal anastomoses in the 3 coronary artery territories were similar between the 2 groups. The mean flows of the left ITA proximal to the Y-anastomosis and of the SV conduit distal to the Y-anastomosis measured according to transit time flow measurement were also similar between the 2 groups. The Jackson-Pratt drain was removed 5 days (median; interquartile range, 3.5-6.5) postoperatively in group LLV and 5 days (median; interquartile range, $3.5-11)$ postoperatively in group $\operatorname{ULV}(P=.673$; Table 2$)$.

\section{Histologic and Immunohistochemical Study}

The mean internal luminal diameters of the proximal and dilated proximal SV segments were significantly smaller in the LLV group than in the ULV group (groups LLV vs ULV: proximal segment, $623 \pm 143 \mu \mathrm{m}$ vs $858 \pm 266 \mu \mathrm{m}$; $P=.008$; dilated proximal segments, $1138 \pm 419 \mu \mathrm{m}$ vs $1477 \pm 353 \mu \mathrm{m} ; P=.047$ ), although those of the distal SV segments were not significantly different between the 2 groups (Figure 3,A). The mean luminal diameters of the dilated proximal SV segments, which had been exposed to the native flow and pressure of the left ITA for passive dilation, were significantly larger than those of the adjacent proximal SV segments in both groups (group LLV, $1138 \pm 419 \mu \mathrm{m}$ vs $623 \pm 143 \mu \mathrm{m} ; P=.004$; group ULV, $1477 \pm 353 \mu \mathrm{m}$ vs $858 \pm 266 \mu \mathrm{m} ; P<.001)$.

The mean intimal thickness of the distal, proximal, and dilated proximal segments showed no significant differences between the 2 groups, although the dilated proximal segment intima became significantly thinner than the proximal segment intima in both groups (Figure 3, B). The mean medial thickness of the distal, proximal, and dilated proximal segments was not significantly different between the 2 groups, and the dilated proximal segment media showed insignificant changes after dilation in both groups (Figure 3, C). The grades of endothelial integrity, expression of VSMC activation-related proteins, and preservation of medial smooth muscle folding of the proximal and dilated proximal SV segments were not different between the LLV and ULV groups, although significantly lower grades of those parameters were observed in the dilated proximal than in the proximal SV segments in both groups (Table 3). However, KLF4 (proximal) and myocardin (dilated proximal) showed greater than medium $(>0.5)$ effect size, although the $P$ values were larger than .05 . This meant that the difference in those variables might be underestimated because of the small sample size.

\section{Angiographic Results and Clinical Outcomes}

The early postoperative angiographic results showed similar patency rates between the LLV and ULV groups (ITA patency rate: 13 of 13 vs 13 of $13 ; P>.999$; SV patency rate: 34 of 34 vs 33 of 34; $P>.999$ ). One SV anastomosis to the obtuse marginal coronary artery was occluded in the ULV group. The 1-year angiographic results showed that the patency rates were also similar in the LLV and ULV groups (ITA patency rate: 12 of 12 vs 12 of 12; $P>$.999; SV patency rate: 28 of 31 vs 28 of 31 ; $P>$.999). Three SV anastomoses in 2 patients were occluded in each group.

TABLE 2. Surgical data

\begin{tabular}{|c|c|c|c|c|}
\hline & Group LLV $(n=13)$ & Group ULV $(n=13)$ & $P$ value & SMD \\
\hline Length of SV harvested, $\mathrm{cm}$ & $22.1 \pm 1.9$ & $22.4 \pm 2.0$ & $.869 *$ & 0.16 \\
\hline Length of SV used for distal anastomoses, $\mathrm{cm}$ & $17.6 \pm 1.8$ & $18.4 \pm 2.0$ & $.829 *$ & 0.18 \\
\hline \multicolumn{5}{|l|}{ SV harvest site } \\
\hline Left leg & $12(92 \%)$ & $10(77 \%)$ & - & \\
\hline Right leg & $1(8 \%)$ & $3(23 \%)$ & - & \\
\hline \multicolumn{5}{|l|}{ Coronary artery territories revascularized using SV } \\
\hline Left anterior descending coronary artery & 19 & 21 & - & \\
\hline Left circumflex coronary artery & 15 & 13 & - & \\
\hline Right coronary artery & 11 & 12 & - & \\
\hline Total number of distal anastomoses & $3.6 \pm 0.7$ & $3.6 \pm 0.8$ & $.938^{*}$ & $<0.01$ \\
\hline Number of distal anastomoses per the SV & $2.6 \pm 0.7$ & $2.6 \pm 0.7$ & $.938^{*}$ & $<0.01$ \\
\hline Left ITA flow proximal to Y-anastomosis, $\mathrm{mL} / \mathrm{min} \dagger$ & $52 \pm 23$ & $45 \pm 24$ & $.471^{*}$ & 0.27 \\
\hline SV composite graft flow, $\mathrm{mL} / \mathrm{min} \dagger$ & $29 \pm 18$ & $31 \pm 22$ & $.890^{*}$ & 0.07 \\
\hline SV composite graft/left ITA flow ratio & $0.5 \pm 0.2$ & $0.5 \pm 0.2$ & $.129 *$ & \\
\hline
\end{tabular}

$L L V$, Lower leg saphenous vein; $U L V$, upper leg saphenous vein; $S M D$, standardized mean difference; $S V$, saphenous vein; ITA, internal thoracic artery. *Mann-Whitney $U$ test. $\dagger$ Mean flow measured using transit time flow measurement. 

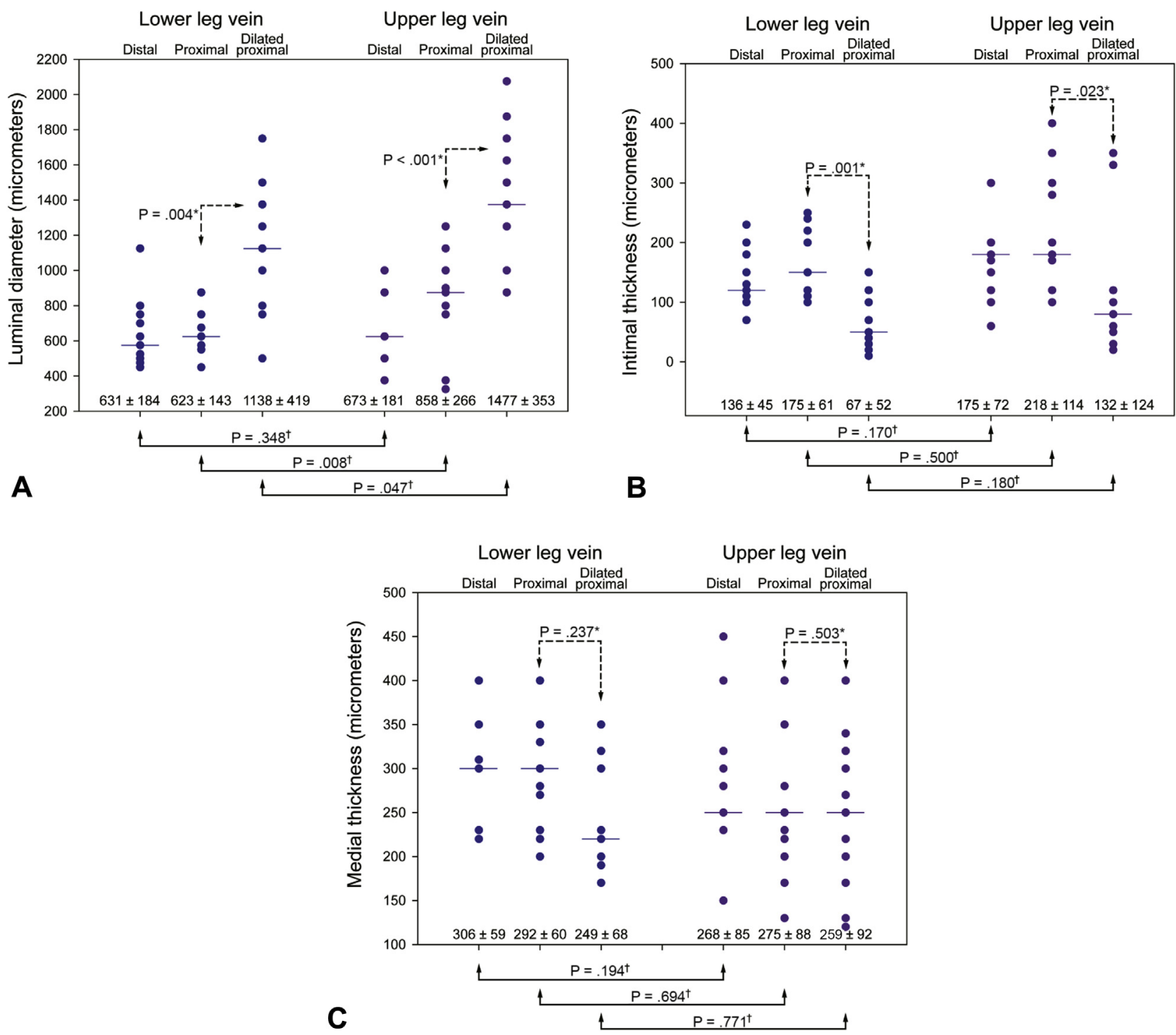

FIGURE 3. Luminal diameter (A), intimal (B), and medial (C) thicknesses of the distal, proximal, and dilated proximal segments in the lower leg and upper leg saphenous vein composite graft groups. Each of the data points was plotted with a bar to indicate the median value. * Wilcoxon signed rank test; $\dagger$ MannWhitney $U$ test.

The mean diameters of the left ITA were significantly larger $(2.8 \pm 0.5 \mathrm{~mm}$ vs $3.2 \pm 0.6 \mathrm{~mm} ; P<.001)$ on the 1 -year angiogram than on the early postoperative angiogram, whereas those of the SV were significantly smaller $(4.4 \pm 0.8 \mathrm{~mm}$ vs $3.0 \pm 1.4 \mathrm{~mm} ; P<.001)$. In the early postoperative as well as 1-year angiograms, the SV mean diameters did not differ between the LLV and ULV groups, although significantly smaller mean diameters of the SV were observed on the 1-year angiogram than on the early postoperative angiogram in both groups (Figure 4, $A$ and $B$, Video 1; a patient's angiograms taken at different times).

The mean SV filling frame counts were significantly lower ( $31 \pm 12$ vs $23 \pm 10 ; P=.001$ ) on the 1-year angiogram than on the early postoperative angiogram. On the early postoperative as well as 1-year angiograms, the mean SV filling frame counts were not different between the LLV and ULV groups, although significantly lower mean SV filling frame counts on the 1-year angiogram than those on the early postoperative angiogram were observed in both groups (Figure 4,C). There was no inhospital mortality, but 1 patient in the ULV group died of sepsis after colon surgery 4 months after CABG.

\section{DISCUSSION}

The present study showed 3 main findings. First, the intimal and medial thicknesses and immunohistochemical findings were not significantly different between the lower versus upper leg "no-touch" SV conduits, although the 
TABLE 3. The degrees of saphenous vein endothelial integrity (hematoxylin and eosin), expression of vascular smooth muscle cell activationrelated proteins (Krüppel-like factor 4 and serum response factor), and preservation of medial smooth muscle folding (myocardin)

\begin{tabular}{|c|c|c|c|c|}
\hline & Group LLV $(\mathrm{n}=13)$ & Group ULV $(n=13)$ & $P$ value & SMD \\
\hline \multicolumn{5}{|l|}{ Hematoxylin and eosin } \\
\hline Proximal & $0.76 \pm 0.13$ & $0.78 \pm 0.15$ & $>.999 *$ & 0.11 \\
\hline Dilated proximal & $0.36 \pm 0.23$ & $0.34 \pm 0.16$ & $.967^{*}$ & 0.12 \\
\hline Intragroup pairwise comparison & $P<.001 \dagger$ & $P<.001 \dagger$ & & \\
\hline \multicolumn{5}{|l|}{ Krüppel-like factor 4} \\
\hline Proximal & $0.75 \pm 0.13$ & $0.66 \pm 0.15$ & $.155^{*}$ & 0.61 \\
\hline Dilated proximal & $0.30 \pm 0.21$ & $0.25 \pm 0.11$ & $.843^{*}$ & 0.32 \\
\hline Intragroup pairwise comparison & $P<.001 \dagger$ & $P<.001 \dagger$ & & \\
\hline \multicolumn{5}{|l|}{ Serum response factor } \\
\hline Proximal & $0.72 \pm 0.17$ & $0.63 \pm 0.18$ & $.354^{*}$ & 0.47 \\
\hline Dilated proximal & $0.31 \pm 0.14$ & $0.24 \pm 0.14$ & $.169 *$ & 0.49 \\
\hline Intragroup pairwise comparison & $P<.001 \dagger$ & $P<.001 \dagger$ & & \\
\hline \multicolumn{5}{|l|}{ Myocardin } \\
\hline Proximal & $0.78 \pm 0.21$ & $0.80 \pm 0.13$ & $>.999 *$ & 0.13 \\
\hline Dilated proximal & $0.29 \pm 0.16$ & $0.22 \pm 0.09$ & $.238^{*}$ & 0.53 \\
\hline Intragroup pairwise comparison & $P<.001 \dagger$ & $P<.001 \dagger$ & & \\
\hline
\end{tabular}

$L L V$, Lower leg saphenous vein; $U L V$, upper leg saphenous vein; $S M D$, standardized mean difference. *Mann-Whitney $U$ test. $\dagger$ Wilcoxon signed rank test.

luminal diameters of the proximal and dilated proximal segments of the lower leg SV conduit were significantly smaller on histologic study. Second, significantly larger luminal diameters, thinner intima, and lower grades of immunohistochemical findings were observed in the dilated proximal than in the proximal SV segments in both groups.
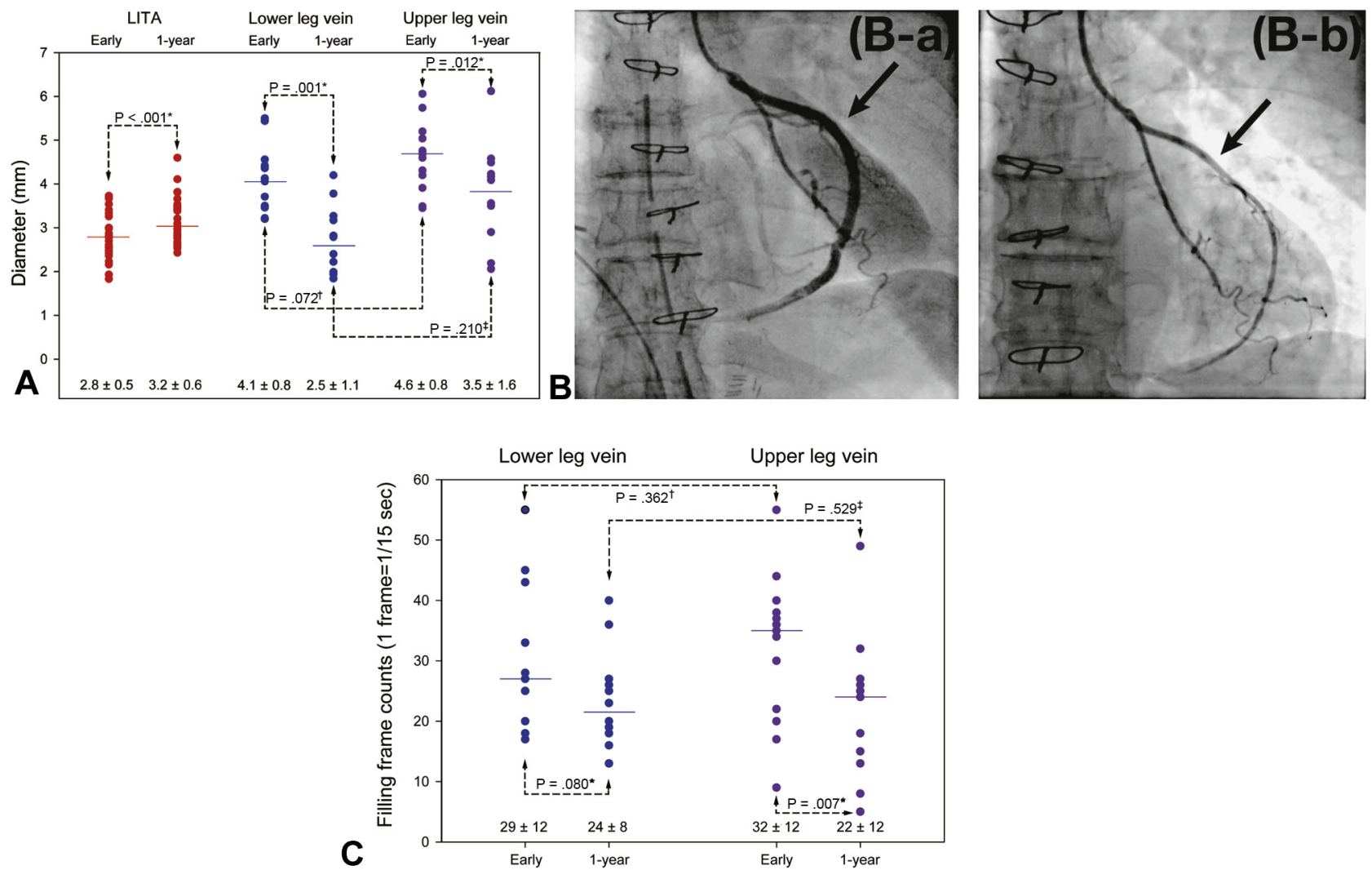

FIGURE 4. A, Conduit diameters on early and 1-year postoperative angiograms. ${ }^{*} P$ value from an analysis of covariance, adjusting for the postoperative vein diameter. B, Patent lower leg saphenous vein (black arrow) composite graft on early $(B-a)$ and 1-year $(B-b)$ postoperative angiograms taken for a patient. C, Filling frame counts of the saphenous vein composite grafts. In (A) and (C), each of the data points were plotted with a bar to indicate the median. LITA, Left internal thoracic artery. *Wilcoxon signed rank test; $\nmid$ Mann-Whitney $U$ test; łanalysis of covariance, adjusting for early postoperative values. 


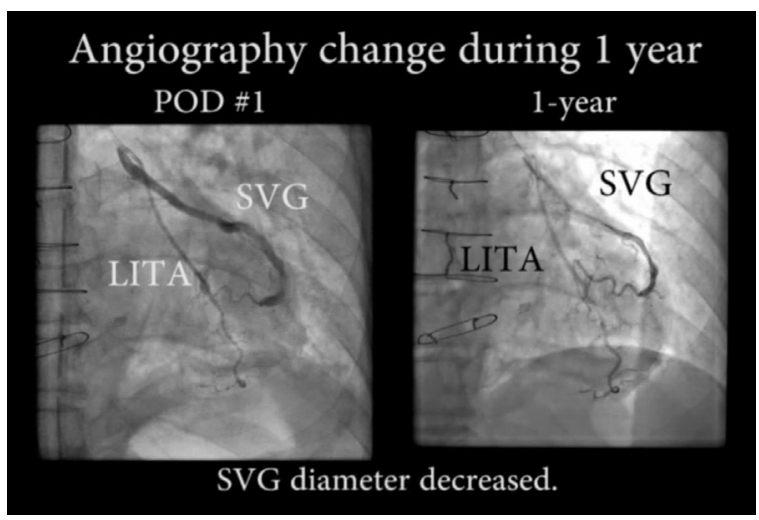

VIDEO 1. The first half of this video shows an example of a decreased saphenous vein diameter during 1-year follow-up. The other half of this video shows an example of increased saphenous vein flow velocity during the same period. Video available at: https://www.jtcvs.org/article/S00225223(18)30939-5/fulltext.

Third, the diameters and patency rates of the "no-touch" SV were not different between the LLV and ULV groups on the 1-year angiogram, although significantly smaller diameters of the SV were observed in both groups.

The great SV is located within the subcutaneous tissue layer in the lower leg. Continuing through the thigh, the SV merges with several superficial veins, becomes larger in diameter and enters the deeper tissues of the upper thigh. The larger upper leg SV is easy to handle; however, this portion of the vein might have a size discrepancy with the target coronary artery or left ITA, and its location might require deeper tissue dissection and cause wound complications. Although there are differences in vessel diameter and localization between the lower and upper leg SV, only a few studies have evaluated functional or morphologic differences. ${ }^{5,9,10}$ One previous study showed more focal hyperplasia and circular muscle hypertrophy and less compliance in the lower leg SV than in the upper leg SV, suggesting less favorable patency of the lower leg SV compared with the upper leg SV. ${ }^{9}$ In contrast, another study showed no significant difference in smooth muscle cell/extracellular matrix scores of smooth muscle layers between conduits derived from the lower and upper leg SV and failed to confirm the higher degree of focal hyperplasia and circular muscle hypertrophy in the lower leg SV. ${ }^{10}$ Another study also showed no significant differences in response to vasoconstrictors or vasodilators or the smooth muscle cell/extracellular matrix ratio between the lower and upper leg SV conduits, suggesting there is no advantage in using one region of the SV over the other as a conduit in CABG. ${ }^{11}$ Our results also suggested no advantage to using either the lower or upper leg SV over the other as a conduit in CABG.

\section{CONCLUSIONS}

There have been various efforts to overcome the structural and functional limitations of using the $\mathrm{SV}$ as a bypass conduit, and studies from our group have shown that the improved SV harvesting technique and the surgical strategy of using the SV as part of a composite graft preserve endothelial structure and function of the SV and result in good SV patency rates despite the inevitable drawbacks of the $\mathrm{SV}$ as a bypass conduit. ${ }^{4-6,8}$ A recent study showed further improved 1-year patency rates of "no-touch" SV composite grafts compared with "minimally manipulated" SV composite grafts $(95.9 \%$ vs $90.0 \% ; P=.035){ }^{8}$

There are several limitations to the present study that must be recognized. First, this prospective, randomized study included a relatively small sample size, although the sample size was statistically calculated on the basis of a previous study. The sample size, with the exception of the primary end point, was calculated by estimating the general measurement results, which might be underpowered for other end points. Two variables (KLF4 [proximal] and myocardin [dilated proximal]) showed effect size (SMD) greater than medium $(>0.5)$ effect size, which meant that the difference between groups might be underestimated because of the small sample size. Second, the lower leg or upper leg SV conduit was harvested in each patient. We did not compare the lower versus upper leg SV conduit in the same patient because revascularization using a lower or upper leg SV composite graft was sufficient for complete revascularization in most patients with 3-vessel disease. Third, a long-term angiographic study with a large patient population might be necessary for a definitive conclusion regarding the advantage of using either a lower or an upper leg SV conduit.

Although the proximal segment luminal diameters were smaller in the lower leg SV, there were no differences in the immunohistochemical results or patency rates on early and 1-year postoperative angiograms between the lower and upper leg "no-touch" SV conduits.

\section{Conflict of Interest Statement}

Authors have nothing to disclose with regard to commercial support.

\section{References}

1. Lytle BW, Loop FD, Cosgrove DM, Ratliff NB, Easley K, Taylor PC Long-term (5 to 12 years) serial studies of internal mammary artery and saphenous vein coronary bypass grafts. J Thorac Cardiovasc Surg. 1985;89:248-58.

2. Loop FD, Lytle BW, Cosgrove DM, Stewart RW, Goormastic M, Williams GW, et al. Influence of the internal-mammary-artery graft on 10-year survival and other cardiac events. N Engl J Med. 1986;314:1-6.

3. Kim KB, Cho KR, Jeong DS. Midterm angiographic follow-up after off-pump coronary artery bypass: serial comparison using early, 1-year, and 5-year postoperative angiograms. J Thorac Cardiovasc Surg. 2008; 135:300-7.

4. Hwang HY, Kim MA, Seo JW, Kim KB. Endothelial preservation of the minimally manipulated saphenous vein composite graft: histologic and immunohistochemical study. J Thorac Cardiovasc Surg. 2012;144:690-6.

5. Kim KB, Hwang HY, Hahn S, Kim JS, Oh SJ. A randomized comparison of the Saphenous Vein Versus Right Internal Thoracic Artery as a Y-Composite Graft 
(SAVE RITA) trial: one-year angiographic results and mid-term clinical outcomes. J Thorac Cardiovasc Surg. 2014;148:901-7; discussion: 907-8.

6. Hwang HY, Lee KH, Han JW, Kim KB. Equivalency of saphenous vein and arterial composite grafts: 5-year angiography and midterm clinical follow-up. Ann Thorac Surg. 2016;102:580-8.

7. Samano N, Geijer H, Liden M, Fremes S, Bodin L, Souza D. The no-touch saphenous vein for coronary artery bypass grafting maintains a patency, after 16 years, comparable to the left internal thoracic artery: a randomized trial. $J$ Thorac Cardiovasc Surg. 2015;150:880-8.

8. Kim YH, Oh HC, Choi JW, Hwang HY, Kim KB. No-touch saphenous vein harvesting may improve further the patency of saphenous vein composite grafts: early outcomes and 1-year angiographic results. Ann Thorac Surg. 2017;103:1489-97.

9. Davies AH, Magee TR, Baird RN, Sheffield E, Horrocks M. Vein compliance: a preoperative indicator of vein morphology and of veins at risk of vascular graft stenosis. Br J Surg. 1992;79:1019-21.

10. Stooker W, Gok M, Sipkema P, Niessen HW, Baidoshvili A, Westerhof N, et al. Pressure-diameter relationship in the human greater saphenous vein. Ann Thorac Surg. 2003;76:1533-8.
11. Golbasi I, Tasatargil A, Aksoy NH, Sadan G, Karasu E, Turkay C, et al. A functional and histopathological comparison - of proximal and distal saphenous vein contractility and morphology. Tex Heart Inst J. 2005;32:287-93.

12. Rennie D. How to report randomized controlled trials: the CONSORT statement. JAMA. 1996;276:649.

13. Souza DS, Johansson B, Bojo L, Karlsson R, Geijer H, Filbey D, et al. Harvesting the saphenous vein with surrounding tissue for $\mathrm{CABG}$ provides long-term graft patency comparable to the left internal thoracic artery: results of a randomized longitudinal trial. J Thorac Cardiovasc Surg. 2006;132:373-8.

14. Wanjare M, Kusuma S, Gerecht S. Perivascular cells in blood vessel regeneration. Biotechnol J. 2013;8:434-47.

15. Cohen J. The T test for means. In: Cohen J. Statistical Power Analysis for the Behavioral Science. 2nd ed. New York: Lawrence Erlbaum Associates Publishers, 1988; 20-7.

Key Words: saphenous vein, coronary artery bypass grafting, anatomy, patency

Readers who found these articles interesting may also like to read the following papers found in recent and future issues of our sister publications, Seminars in Thoracic and Cardiovascular Surgery and Operative Techniques in Thoracic and Cardiovascular Surgery!

\section{Adult: Coronary}

ORIGINAL SUBMISSION: Remote Ischemic Preconditioning in High Risk Cardiovascular Surgery Patients: A RandomizedControlled Trial. Nicole S. Coverdale. Semin Thoracic Surg 2018: 26-33

Editorial Commentary: Remote Ischemic Preconditioning: A Complex Question with an Even More Complex Answer. Irving L. Kron. Semin Thoracic Surg 2018: 34-35 
$H \& E$
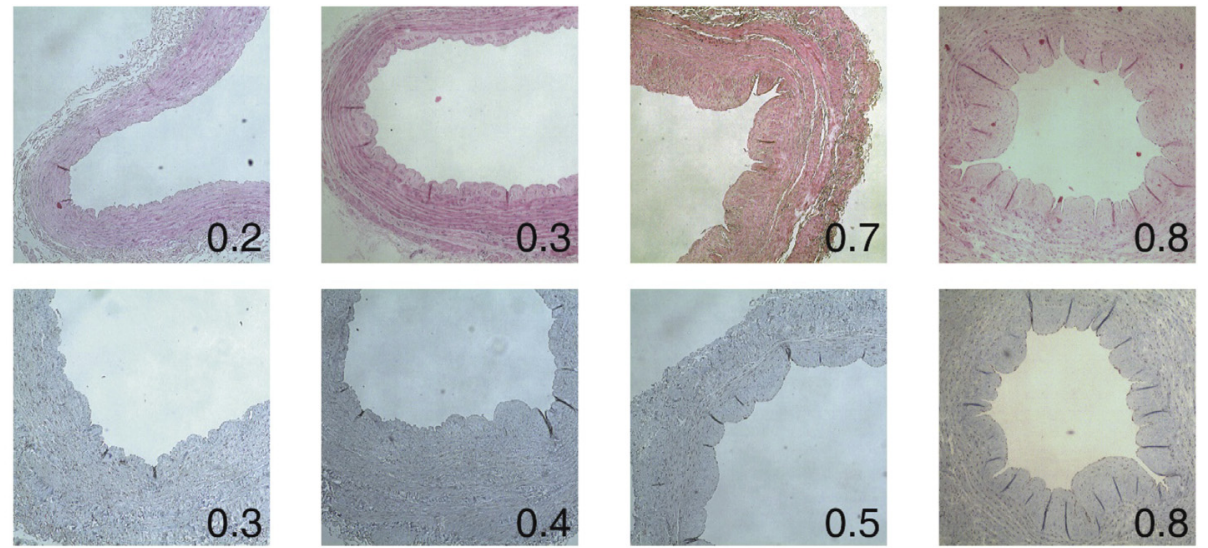

KLF4
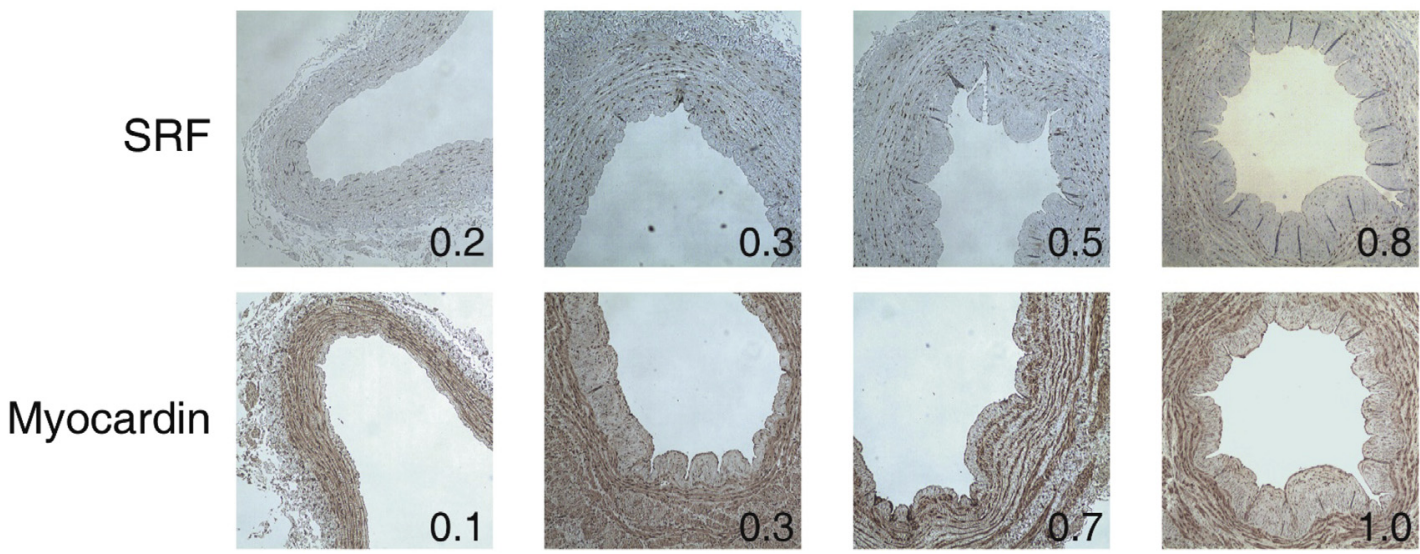

FIGURE E1. Representative examples of specimen; endothelial integrity (H\&E), expression of vascular smooth muscle cell (VSMC) activation-related proteins (KLF4 and SRF), and preservation of smooth muscle folding (myocardin). Numbers indicate degree of measured variables calculated according to the proportion of positive staining in luminal circumference (between 0 and 1). $H \& E$, Hematoxylin and eosin; $K L F 4$, Krüppel-like factor 4; $S R F$, serum response factor.

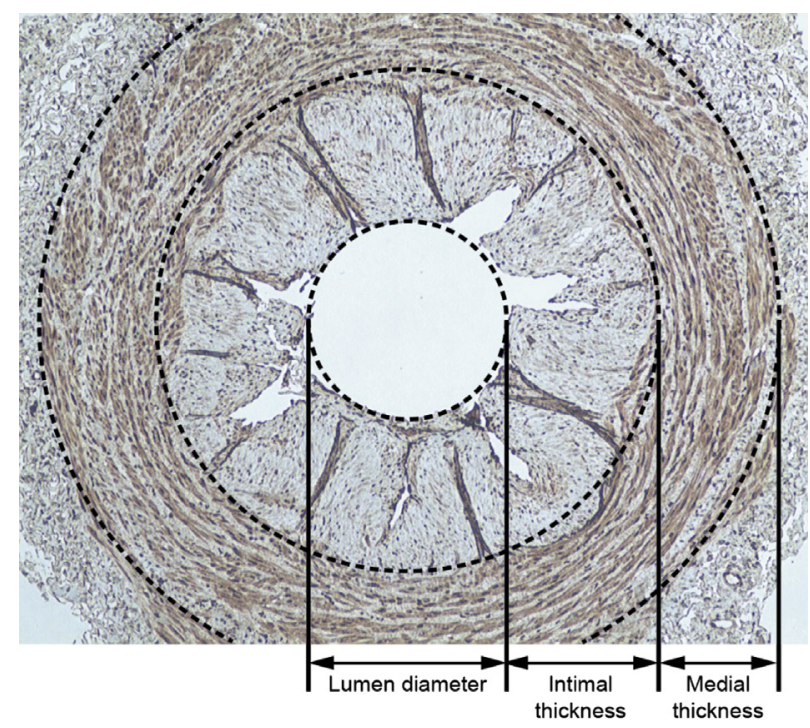

FIGURE E2. A BX51 optical microscope (Olympus Corp, Tokyo, Japan) was used for the microscopic examination, and the internal luminal diameter as well as the intimal and medial thicknesses were measured using a U-OCM10/100 eyepiece micrometer (Olympus Corp) and a stage micrometer (Narika Corp, Tokyo, Japan). 


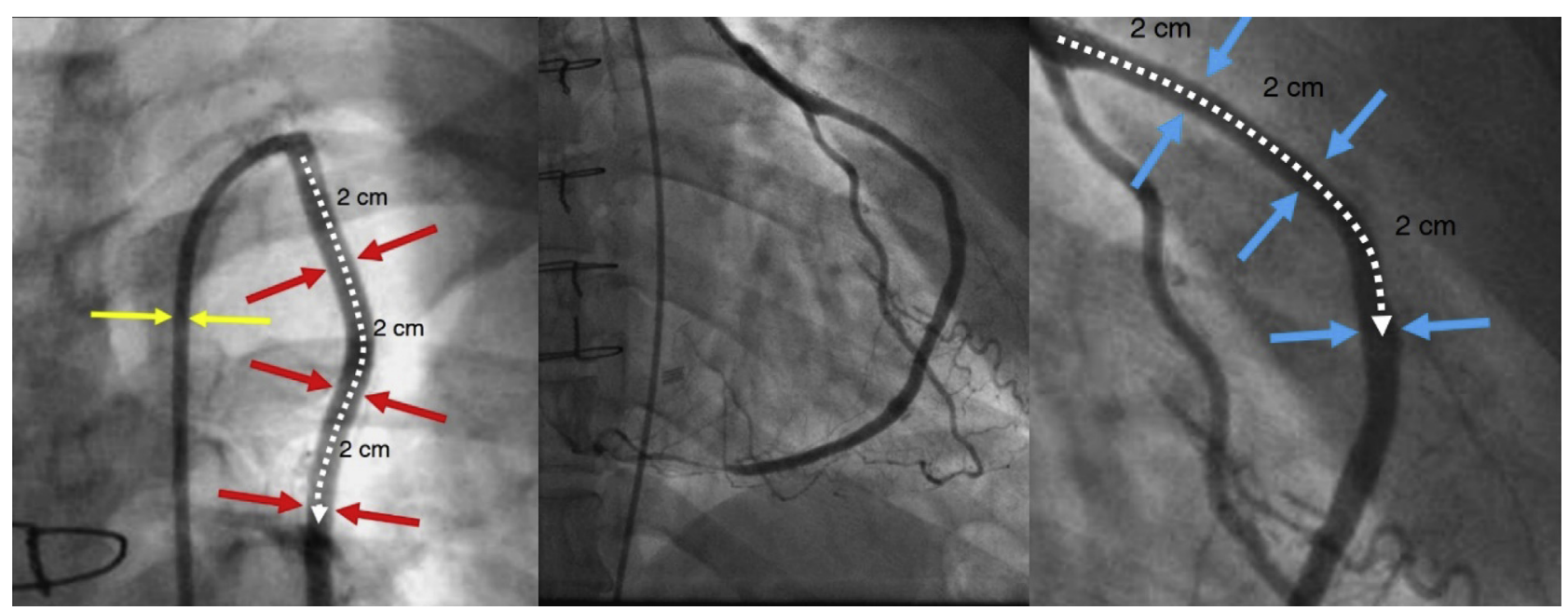

FIGURE E3. Measuring internal luminal diameter from angiogram. A single experienced observer measured the lumen diameters of conduits using the guiding catheter (yellow arrow) for calibration and the scientific image analysis software ImageJ (National Institute of Health, Bethesda, Md). The left internal thoracic artery diameters were measured at 3 points $(2,4$, and $6 \mathrm{~cm}$ distal to the left internal thoracic artery origin, red arrows), and the average value was calculated. The diameters of the saphenous vein conduits were also measured at 3 points $(2,4$, and $6 \mathrm{~cm}$ distal to the Y-anastomosis, blue arrows), and the average value was calculated. 